\title{
SEQUÍAS EN CHILE CENTRAL A PARTIR DE DIFERENTES ÍNDICES DESDE 1824
}

\author{
Pablo SARRICOLEA ${ }^{1,3}$, Oliver MESEGUER-RUIZ ${ }^{2,3}$ \\ ${ }^{1}$ Departamento de Geografia. Universidad de Chile. \\ ${ }^{2}$ Departamento de Ciencias Históricas y Geográficas. Universidad de Tarapacá. \\ ${ }^{3}$ Grupo de Climatología, Universidad de Barcelona. \\ psarricolea@uchilefau.cl, omeseguer@uta.cl
}

\section{RESUMEN}

Se analizan las sequías meteorológicas en Chile central con el propósito de caracterizar su ocurrencia en múltiples escalas temporales, y encontrar indicios de cambios en su variabilidad y frecuencia. Para ello se ha dispuesto de 27 estaciones meteorológicas a resolución diaria (1981-2010), las cuales permiten identificar períodos secos bajo el umbral 0,1 mm. Además, se establece la longitud de las sequías. Se aplicó el índice estándar de precipitación (SPI) para conocer la variabilidad de la sequía. En el caso de Quinta Normal se estimó las megasequías desde 1824. Los resultados indican mayor persistencia de días secos en la región de Valparaíso, lo cual es consistente con la longitud de las rachas de sequía, en muchos casos superior a 22 días al año. El evento La Niña en el invierno de 1998 provocó la mayor racha de días secos del trimestre lluvioso (67 días), sumando en total 86 días sin lluvia en Quinta Normal. Las megasequías no poseen un patrón claro, pero dada la reducción de la precipitación se harán más frecuentes.

Palabras clave: Chile central, clima mediterráneo, índice de rachas, precipitación diaria, megasequía.

\begin{abstract}
Meteorological droughts in Central Chile have been analyzed with the purpose of characterize its occurrence in multiple time scales and find evidence of changes in its variability and frequency. Twenty seven meteorological stations with a daily resolution (1981-2010) have been used to identify dry periods under a $0.1 \mathrm{~mm}$ threshold. Furthermore, the length of droughts is established. The Standard Precipitation Index (SPI) is applied to know the drought variability. In the case of Quinta Normal, the megadroughts since 1824 are considered. Results show a higher persistence of dry days in the Valparaíso region, which is consistent with the drought spell lengths, in many cases higher than 22 days each year. La Niña event in the 1998 winter induced the longer dry days spell of the rainy three-month period, with 86 days without rain in Quinta Normal. Megadroughts do not have a clear pattern, but according to the precipitation reduction, they'll be more frequent.
\end{abstract}

Key words: Central Chile, Mediterranean climate, spell index, daily precipitation, megadrought.

\section{INTRODUCCIÓN}


La sequía es un extremo hidrometeorólogico de efectos acumulativos y desastrosos, afectando a la sociedad y los sistemas físicos naturales. Posee una extensión temporal distinta a otros extremos hidrometeorológicos, pues, mientras que la precipitación abundante se registra en pocas horas o días, las sequías pueden afectar un territorio durante meses o años.

La sequía representa un problema de múltiples dimensiones: afecta a la economía, los sistemas ecológicos, la sociedad, generación de energía, etc. Debido a ello, la sequía presenta diversas definiciones, las cuales, en algunos casos, van más allá de la propia condición de ausencia de lluvia o sequía meteorológica.

Según Below et al. (2007), entre 1900 y 2004 la sequía fue el riesgo natural que provocó las mayores pérdidas humanas (54\%), afectando a un tercio de la población mundial (sólo detrás de inundaciones, que agrupa al 51\%) y ocupando el cuarto lugar en las pérdidas económicas, las cuales son lideradas por los huracanes, inundaciones $\mathrm{y}$ terremotos.

El interés de estudiar la sequía en Chile central (región de Valparaíso y Metropolitana) se debe a que en ella se emplaza gran parte de las actividades demandantes de agua (agricultura, minería y los centros poblados), por lo que la disponibilidad de agua es un factor estratégico para el desarrollo. El área de estudio presenta una alta vocación de producción agrícola y vitivinícola, lo que redunda en una fragilidad ante el desastre de la sequía. Desde 2007 esta región es afectada por una de las peores sequías que se tenga registro, la cual ha sido denominada como "Megasequía" (Boisier et al. 2016). Es más, se proyecta que serán cada vez más frecuentes. En Chile central en el contexto de cambio climático se proyecta escenarios de alzas de las temperaturas de $2^{\circ}$ a $4^{\circ} \mathrm{C}$ y disminuciones cercanas al $30 \%$ en los montos de precipitación (Fuenzalida et al. 2007, Bravo et al. 2014).

Los períodos secos y lluviosos en Chile central están asociados a los centros de acción dominantes en superficie, en este caso el Anticiclón del Pacífico Sur Oriental (APSO) y el Cinturón de Bajas Presiones Subpolares (CBPP), más las configuraciones en altura (vaguadas, dorsales, altas y bajas segregadas) (Sarricolea y Meseguer-Ruíz, 2015). A ellas se debe sumar el efecto sobre las precipitaciones que confieren los patrones de variabilidad de baja frecuencia. Para Chile central se distinguen El Niño Oscilación del Sur (ENSO), la Oscilación Decadal del Pacífico (PDO) y la Oscilación Antártica (AAO) (Sarricolea et al., 2014).

Según Bravo et al. (2014), los modelos del IPCC (2013) aplicados a Chile central (en su escenario más pesimista RCP8.5) señalan que se podrían triplicar la frecuencia de las sequías, y con ello las de longitud temporal bienales y de 3 años o más. Con esto, la sequía adquiere una importancia fundamental en las actividades económicas que se desarrollan en esta región, al igual que el abastecimiento de agua para consumo.

\section{MÉTODOS}

El área de estudio abarca administrativamente la V Región y la XIII Región, correspondientes a Valparaíso y Metropolitana. Estas regiones poseen una ubicación geográfica aproximada entre $\operatorname{los} 32^{\circ} 01^{\prime}$ y los $34^{\circ} 08^{\prime}$ de latitud Sur y entre los $71^{\circ} 45^{\prime}$ y $69^{\circ} 46^{\prime}$ de longitud Oeste, y una superficie de $31.387,02 \mathrm{Km}^{2}$. Posee un clima tran- 
sicional entre el Semiárido y el Mediterráneo, una alta concentración de población y actividades productivas, las cuales demandan intensivamente agua, y son afectadas por anomalías climáticas de distinta naturaleza, en especial por fases de diferente signo de teleconexiones, tales como ENSO, PDO y AAO, las cuales modulan períodos secos y lluviosos.

En total se han utilizado 27 estaciones meteorológicas (ver Tabla 1), las cuales se sitúan entre los 25 y 2.575 metros sobre el nivel del mar. Todas ellas cubren el período 1981-2010. Su localización espacial se puede ver en la Fig. 1.

\begin{tabular}{|c|c|c|c|c|c|}
\hline $\mathbf{N}^{\circ}$ & Estación & $\begin{array}{l}\text { Latitud } \\
\text { (S) }\end{array}$ & $\begin{array}{l}\text { Longitud } \\
\text { (W) }\end{array}$ & Altitud (m) & Fuente \\
\hline 1 & Pedernal & $-32,09$ & $-70,80$ & 1100 & DGA \\
\hline 2 & Quilimarí & $-32,12$ & $-71,50$ & 25 & DGA \\
\hline 3 & Chalaco & $-32,19$ & $-70,79$ & 880 & DGA \\
\hline 4 & El Trapiche & $-32,23$ & $-70,71$ & 1180 & DGA \\
\hline 5 & Palquico & $-32,25$ & $-71,14$ & 450 & DGA \\
\hline 6 & Alicahue & $-32,34$ & $-70,75$ & 750 & DGA \\
\hline 7 & Estero Rabuco & $-32,85$ & $-71,12$ & 300 & DGA \\
\hline 8 & Vilcuya & $-32,86$ & $-70,47$ & 1100 & DGA \\
\hline 9 & Riecillos & $-32,93$ & $-70,35$ & 1290 & DGA \\
\hline 10 & Caleu & $-33,01$ & $-70,99$ & 1150 & DGA \\
\hline 11 & Embalse Rungue & $-33,02$ & $-70,91$ & 700 & DGA \\
\hline 12 & Valparaíso & $-33,06$ & $-71,61$ & 41 & $\mathrm{DMC}$ \\
\hline 13 & Lago Peñuelas & $-33,15$ & $-71,54$ & 360 & DGA \\
\hline 14 & Colliguay & $-33,17$ & $-71,15$ & 490 & DGA \\
\hline 15 & Cerro Calán & $-33,40$ & $-70,54$ & 800 & DGA \\
\hline 16 & Quinta Normal & $-33,41$ & $-70,79$ & 527 & $\mathrm{DMC}$ \\
\hline 17 & Los Panguiles & $-33,44$ & $-71,03$ & 195 & DGA \\
\hline 18 & Terrazas DGA & $-33,45$ & $-70,65$ & 560 & DGA \\
\hline 19 & Antupirén & $-33,50$ & $-70,52$ & 920 & DGA \\
\hline 20 & San Antonio & $-33,57$ & $-71,62$ & 150 & DGA \\
\hline 21 & Cerrilos de Leyda & $-33,63$ & $-71,51$ & 180 & DGA \\
\hline 22 & San José de Maipo & $-33,64$ & $-70,35$ & 970 & DGA \\
\hline 23 & Pirque & $-33,67$ & $-70,59$ & 670 & DGA \\
\hline 24 & Embalse El Yeso & $-33,68$ & $-70,09$ & 2575 & DGA \\
\hline 25 & Melipilla & $-33,68$ & $-71,20$ & 170 & DGA \\
\hline 26 & Carmen de las Rosas & $-33,76$ & $-71,15$ & 165 & DGA \\
\hline 27 & San Gabriel & $-33,78$ & $-70,24$ & 1240 & DGA \\
\hline
\end{tabular}

Tabla 1: Estaciones meteorológicas consideradas, según localización y fuente de origen. 


\subsection{Método de rachas}

Se trata de hallar la longitud de las rachas secas, es decir, de las secuencias de días secos consecutivos, del período de estudio (Martín-Vide, 2003). La aplicación de este método consiste en contabilizar el número absoluto de rachas o secuencias de días secos durante el período de estudio, así como cuantificar la longitud promedio, máxima y mínima absoluta de las mismas. Para ello, previamente se deben haber agrupado las jornadas de días secos en rachas. El cálculo de la longitud media de las rachas de sequía $\left(l_{1}\right)$ se obtiene como cociente entre el total de días de secos (L) y el total de rachas (NR) del período de estudio considerado.

$$
l_{1}=L / N R
$$

\subsection{EI Índice Estándar de Precipitación (SPI)}

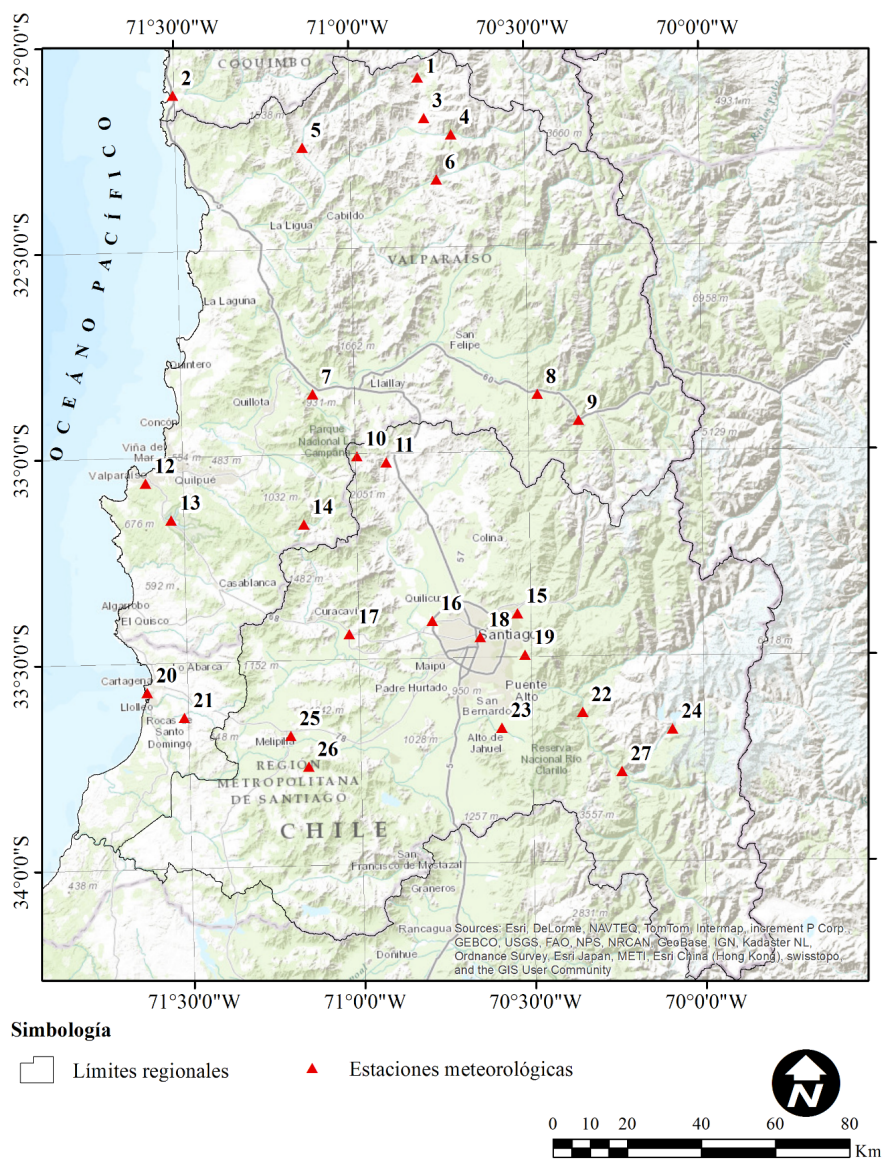

Fig. 1: Localización de las estaciones meteorológicas en el área de estudio. Los números aparecen en la Tabla 1. Fuente: Sarricolea y Meseguer-Ruiz, 2015 
Mejorar la detección del comienzo de la sequía y para realizar su seguimiento (Mckee et al. 1993). Debido a que las sequías tienen una gran variación en su duración, es importante detectarlas y monitorizarlas en una amplia variedad de escalas temporales. Las sequías de corto término son medidas por instrumentos meteorológicos y son definidas de acuerdo a la climatología local normal. Las sequías de importancia para la agricultura señalan déficit de la humedad del suelo, siendo sus mejores representantes aquellas de tres a seis meses. Las sequías más prolongadas (de semestres a años) pueden alcanzar impactos significativos sobre las reservas de agua, tanto superficial y subterránea. Los valores de SPI se obtienen mediante la relación estandarizada de las precipitaciones durante un intervalo de tiempo específico (promedios de 1, 3 o 6 meses), es decir, se usan puntajes Z, idealmente de distribuciones Gumbel.

Para alcanzar una mejor representación espacial de las rachas se utilizan técnicas de regresión lineal múltiple, basada en variables tales como la latitud, longitud, altitud, precipitación anual. Los resultados cartográficos fueron elaborados en Sistema de Información Geográfica (SIG) ArcGis 10.2. Finalmente, a los datos de Quinta Normal desde 1824-2015 se aplicarlos los test de homogeneidad de Von Neumann y Thom.

\section{RESULTADOS}

Respecto a los extremos de rachas medias de sequía, se tiene que ellas fluctúan entre 14 y 32 días (Embalse El Yeso y Quilimarí), siendo muy dependientes del gradiente latitudinal y altitudinal. La confección de la cartografía fue mediante una regresión líneal múltiple, la cual alcanzó un coeficiente de correlación de $0,84\left(\mathrm{R}^{2}=0,70\right)$, siendo los valores estadísticamente significativos (p-valor igual a 0,000 ), usando tres variables: altitud (metros), latitud (grados decimal negativos) y precipitación anual (milímetros). La altitud corresponde al valor de cada píxel de la misión topográfica radar Shutlle (Shuttle Radar Topography Mission) o SRTM de resolución de 90 metros, y la precipitación anual a la modelada por Pliscoff et al. (2014).

Rachas de sequía=250,063-0,002×Altitud+6,990×Latitud -0,006×Pp Anual

Los resultados de la ecuación implican que, por ejemplo, el incremento de la latitud en un grado disminuye la longitud de la sequía en 7 días, o que aumentar la altitud en 500 metros la disminuye en un día, siendo la precipitación anual la variable menos sensible, pues 100 milímetros más de lluvia suponen medio día menos de sequía. En este sentido, la cantidad de precipitación media parece la variable más compleja de analizar en función de las rachas de sequía, pero, como su importancia en la ecuación es menor e intervienen otras variables (latitud y altitud), se compensan mutuamente.

Al examinar las longitudes máximas de las rachas de sequía para una estación (Quinta Normal), es posible identificar dos antecedentes muy relevantes. En primer lugar, las rachas mayores a 120 días (de verano y otoño) se han presentado en 14 de los 30 años de la serie analizada: 1984, 1986, 1988, 1989, 1990, 1993, 1994, 1999, 2001, 2002, 2004, 2006, 2009 y 2010; lo cual permite afirmar que la última década ha sido más seca que las dos precedentes, aumentando las sequías de 120 días o más de un $40 \%$ a un $60 \%$. En segundo lugar, las sequías mayores de 30 días en invierno han ocurrido en cinco años bien concretos: 1986, 1989, 1993, 1995 y 1998; este último año con dos períodos, que dieron lugar al invierno con menos días de lluvia (4 en total y 86 días secos), sumando un total de 23,4 $\mathrm{mm}$. Por otra parte, las rachas medias 
muestran las peores cifras en la región de Valparaíso (Fig. 2), pues en promedio superan los 20 días sin lluvia. El gradiente de las rachas de sequía posee una componente latitudinal muy notoria (Fig. 2), indicando una disminución de la longitud de las rachas secas con el aumento de la latitud e incremento altitudinal.

Respecto al SPI de 6 meses se ha encontrado muchas diferencias intra-anuales del comportamiento espacial de la sequía (Fig. 3). Por ejemplo, hay años muy contrastados, eso significa que algunos observatorios marcan alguna categoría de sequía o humedad mientras que el resto de estaciones está en el margen de la "normalidad". Esto confirma que espacialmente esta región posee un importante control topoclimático.

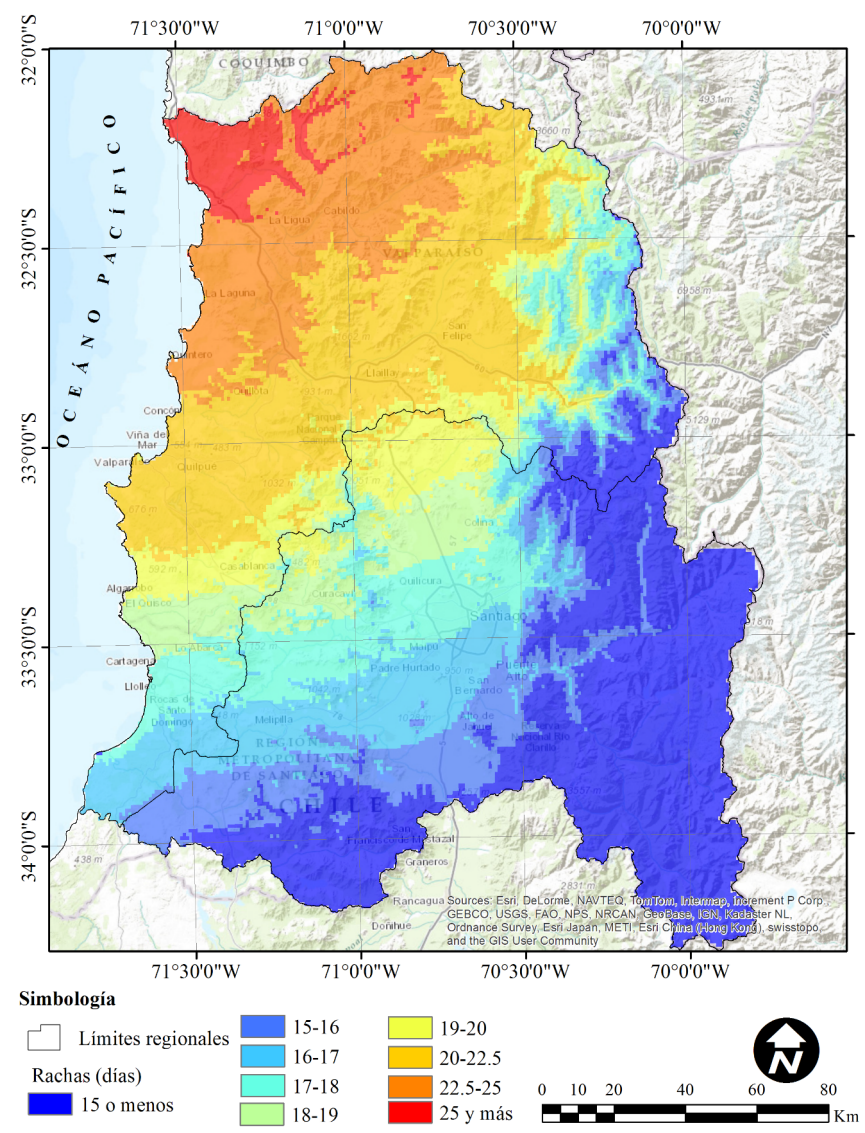

Fig. 2: Mapa de la longitud media de las rachas de sequía. Fuente: Sarricolea y MeseguerRuiz, 2015.

La precipitación de Quinta Normal desde 1824 a 2015 se aprecia en la Fig. 4. Cabe señalar que el observatorio pluviométrico data de 1866, pero según los Anales de la Universidad de Chile (1850), y Vicuña Mackenna (1877) un curioso (Tomás Reyes, empleado de la beneficencia de Santiago) registró datos desde 1824, con lo 
cual se compone una serie de 192 años (Serie homogénea según test de Von Neumann y Thom'. En la Fig. 4 se aprecia la sostenida disminución de la precipitación, desde valores en ventanas móviles de 10 años de $450 \mathrm{~mm}$ montos bajo $250 \mathrm{~mm}$ en la última década, es decir, una caída de $200 \mathrm{~mm}$ en casi dos siglos. Las únicas cuatro sequías comparables con la actual (2007-2015 de promedio de $215 \mathrm{~mm})$ son la de 1869-1876 (212 mm), 1906-1913 (238 mm), 1954-1962 (265 mm) y 1966-1971 (220 mm). De hecho, a decir de Vicuña Mackenna (1877) "Los períodos de humedad del clima han sido más numerosos i frecuentes que los de sequía, especialmente en el presente siglo"

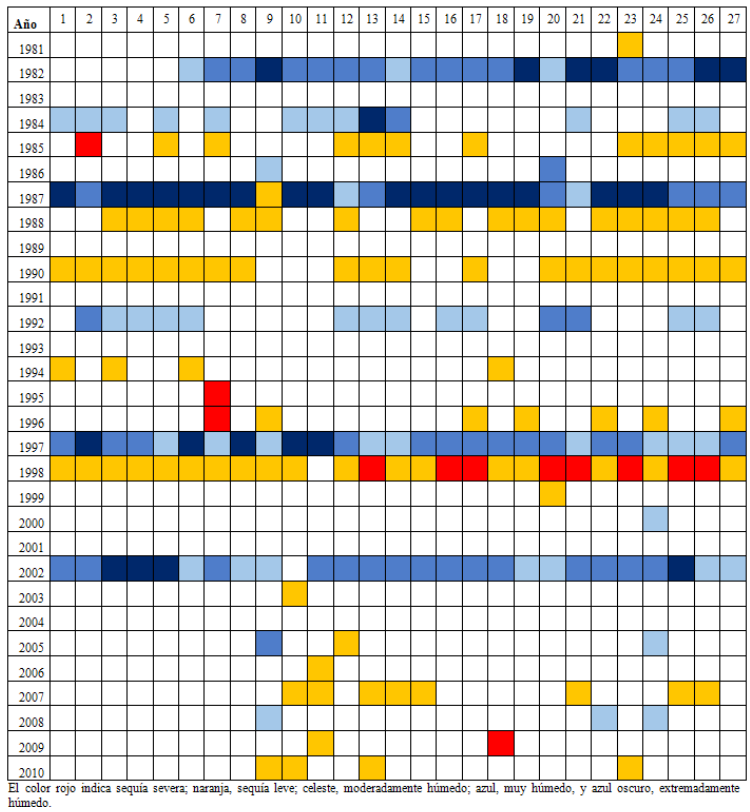

Fig. 3: Índice estandarizado de precipitaciones (SPI) de seis meses expresado en categoría. Fuente: Sarricolea y Meseguer-Ruiz, 2015.

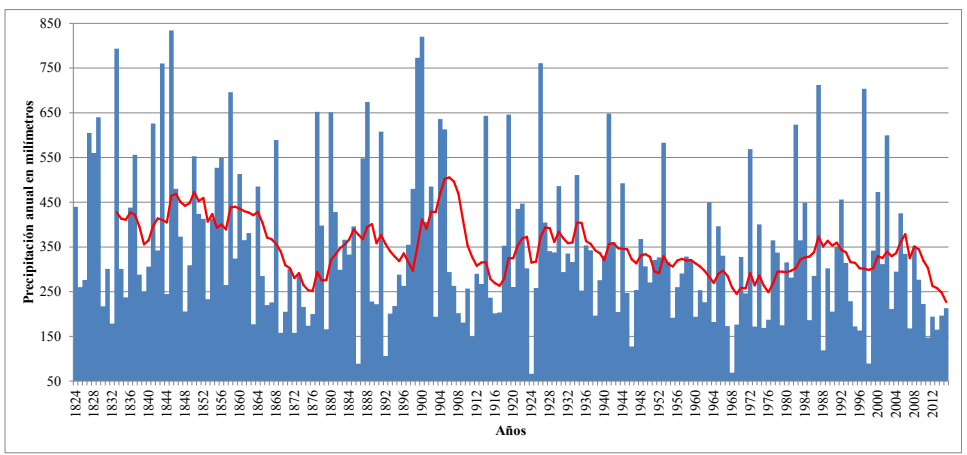

Fig. 4: Precipitación anual de Quinta Normal desde 1824 a 2015. La línea roja representa media móvil de 10 años. Fuente: Elaboración propia. 


\section{DISCUSIÓN Y CONCLUSIONES}

La distribución espacial de las rachas de sequía son de gran ayuda para definir territorios (subcuencas, áreas agroclimáticas y los terrenos de cultivo) que son más afectados por episodios adversos de falta de precipitación, y podrían servir de apoyo a las organizaciones de usuarios de agua, para enfrentar de mejor manera sus efectos.

Según análisis del índice SPI de 6 meses se puede determinar que entre marzo y agosto, la sequía es leve en un 40\%; y moderada a severa en un $7 \%$ y $3 \%$, respectivamente. Esto se explica a que se posee más precipitación y mayor variabilidad interanual.

Las zonas donde la sequía impacta de menor manera (o son más resistentes) corresponde a la precordillera y la cordillera ubicadas entre las regiones de Valparaíso y Metropolitana. Esto se aprecia en la distribución espacial del SPI mínimos en años La Niña, pues son menos afectados por la sequía. Además, en la distribución espacial del SPI máximo en años El Niño, la precordillera y la cordillera son más lluviosos y deben ser considerados como reservorios de agua. También, esta situación se evidencia en las rachas de sequía, mostrando menores longitudes medias de rachas de sequía en el sector antes mencionado.

Lo más relevante, finalmente, es que después de períodos largos de sequía, como el que se ha manifestado actualmente en el país, toman mayor importancia los aportes de los glaciares y acuíferos para disponer de agua en los períodos de primavera y verano. Es decir, si Chile pierde superficie glaciar o no recarga las napas freáticas, la vulnerabilidad ante la menor disponibilidad de agua va a ser absolutamente insostenible.

\section{AGRADECIMIENTOS}

Al proyecto FONDECYT de Iniciación Nº11130629 del Gobierno de Chile, así como a la Dirección General de Aguas y a la Dirección Meteorológica de Chile por la cesión de los datos. También se agradece el apoyo del proyecto PRECABAL (CGL2011-29263-C02-01) del Ministerio de Ciencia e Innovación del Gobierno de España, y del Grupo de Climatología de la Universidad de Barcelona (2014SGR300), así como al Convenio de Desempeño UTA-MINEDUC y al proyecto UTA-Mayor 5744-16.

\section{REFERENCIAS}

Anales de la Universidad de Chile. (1850). Observaciones meteorolójicas de las lluvias i de algunos otros fenómenos análogos, acaecidos en la capital desde el año de 1824 hasta el 1850 especificándose en muchos de ellos las horas de lluvia estando el sol en el horizonte i bajo de él. Anales de la Universidad de Chile. doi:10.5354/0717-8883.1850.1965

Below, R., Grover-Kopec, E., \& Dilley, M. (2007). Documenting drought-related disasters a global reassessment. The Journal of Environment \& Development, 16(3), 328-344.

Boisier, J. P., Rondanelli, R., Garreaud, R. D., \& Muñoz, F. (2016). Anthropogenic and natural contributions to the Southeast Pacific precipitation decline and recent 
mega-drought in central Chile. Geophysical Research Letters, 43(1), 413-421. DOI: $10.1002 / 2015$ GL067265

Bravo, M., Flores, R., Galindo, R., Garreaud, R.D., Muñoz, E., Serey, A., \& Viale, M. (2014). Determinación de posibles impactos en la gestión de los abastecimientos humanos de agua situados en la zona Metropolitana de Chile, provocados por fenómenos asociados a Cambio Climático. Aquaea Paper N5. Fundación Aquaea. $68 \mathrm{pp}$.

Fuenzalida, H., Aceituno, P., Falvey, M., Garreuad, R.D., Rojas, M., \& Sánchez, R. (2007). Study on climate variability for Chile during the 21st century. Technical Report prepared for the National Environmental Committee, Spanish, Santiago.

IPCC. (2013). Climate Change 2013: The Physical Science Basis. Contribution of Working Group I to the Fifth Assessment Report of the Intergovernmental Panel on Climate Change [Stocker, T.F., D. Qin, G.K. Plattner, M. Tignor, S.K. Allen, J. Boschung, A. Nauels, Y. Xia, V. Bex and P.M. Midgley (eds.)]. Cambridge University Press, Cambridge, United Kingdom and New York, NY, USA.

Martín-Vide, J. (2003). El tiempo y el clima. Colección Cuadernos de Medio Ambiente. Rubes Editorial. Barcelona. España.

McKee, T. B., Doesken, N. J., \& Kleist, J. (1995). Drought monitoring with multiple time scales. In Proceedings of the 9th Conference on Applied Climatology (pp. 233-236). Dallas, Boston, MA: American Meteorological Society.

Pliscoff, P., Luebert, F., Hilger, H. H., \& Guisan, A. (2014). Effects of alternative sets of climatic predictors on species distribution models and associated estimates of extinction risk: A test with plants in an arid environment. Ecological Modelling, 288, 166-177.

Sarricolea, P., Meseguer-Ruiz, O., \& Martín-Vide, J. (2014). Variabilidad y tendencias climáticas en Chile central en el período 1950-2010 mediante la determinación de los tipos sinópticos de Jenkinson y Collison. Boletín de la A.G.E. 64: 227-247.

Sarricolea, P., \& Meseguer-Ruiz, O. (2015). Sequías en Chile central a partir de diferentes índices en el período 1981-2010. Investigaciones Geográficas, (50), 19-32.

Vicuña Mackenna, B. (1877). Ensayo histórico del Clima de Chile: :(desde los tiempos prehistóricos hasta el gran temporal de julio de 1877). Imprenta del Mercurio. 